INPLASY

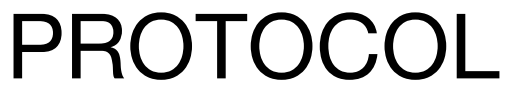

To cite: Zhou et al.

Comparison of cruciateretained and posteriorstabilized implants in total knee arthroplasty: A protocol for systematic review and meta-analysis. Inplasy protocol 202120015. doi:

10.37766/inplasy2021.2.0015

Received: 03 February 2021

Published: 03 February 2021

Corresponding author: Jinwei Guo

ruiying19980618@126.com

Author Affiliation: Jiangjin District Central Hospital

Support: 2016MSXM163.

Review Stage at time of this submission: The review has not yet started.

Conflicts of interest:

None declared.

\section{Comparison of cruciate-retained and posterior-stabilized implants in total knee arthroplasty: A protocol for systematic review and meta-analysis}

Zhou, SM1'; Liu, B²; Guo, JW33.

Review question / Objective: Currently, there are still debates concerning the best implant, whether posterior stabilized or cruciate retaining. This meta-analysis comparing the outcomes of patients undergoing cruciate-retaining versus posterior-stabilized in primary TKA.

Condition being studied: With the posterior cruciate ligament retained, cruciate-retained TKA was thought to be better regarding post-operative knee proprioception and kinesthesia. While others believed that posterior-stabilized TKA had better range of motion, easier in ligament balance, and more reliable femoral rollback. However, there is no systematic review on efficacy and safety of cruciate-retaining versus posterior-stabilized for the treatment of TKA, so our reviewer teams begin to do this job.

INPLASY registration number: This protocol was registered with the International Platform of Registered Systematic Review and Meta-Analysis Protocols (INPLASY) on 03 February 2021 and was last updated on 03 February 2021 (registration number INPLASY202120015).

\section{INTRODUCTION}

Review question / Objective: Currently, there are still debates concerning the best implant, whether posterior stabilized or cruciate retaining. This meta-analysis comparing the outcomes of patients undergoing cruciate-retaining versus posterior-stabilized in primary TKA.
Rationale: With the posterior cruciate ligament retained, cruciate-retained TKA was thought to be better regarding postoperative knee proprioception and kinesthesia. While others believed that posterior-stabilized TKA had better range of motion, easier in ligament balance, and more reliable femoral rollback. However, 
there is no systematic review on efficacy and safety of cruciate-retaining versus posterior-stabilized for the treatment of TKA, so our reviewer teams begin to do this job.

Condition being studied: With the posterior cruciate ligament retained, cruciateretained TKA was thought to be better regarding post-operative knee proprioception and kinesthesia. While others believed that posterior-stabilized TKA had better range of motion, easier in ligament balance, and more reliable femoral rollback. However, there is no systematic review on efficacy and safety of cruciate-retaining versus posteriorstabilized for the treatment of TKA, so our reviewer teams begin to do this job.

\section{METHODS}

Participant or population: TKA patients.

Intervention: Cruciate-retained implant.

Comparator: Posterior-stabilized implants.

Study designs to be included: RCTs.

Eligibility criteria: 2.3.1. Study design Only human RCT studies can be included in the review, while other kinds of study will be excluded, such as observational studies, retrospective analyses, self-controlled trials, case reports, reviews, patient series, animal experiments, etc. 2.3.2. Participants 2.3.2.1. Included population End-staged osteoarthritis patients prepared for TKA. 2.3.2.2. Interventions Treatment group consists of the patients with TKA treated with posterior stabilized implant. Control group consists of the patients with TKA treated with cruciate retaining implant. 2.3.4. Outcomes 2.3.4.1. Primary outcome indicator 1. Knee Society Rating System, clinical (KSCS); 2. functional (KSFS) subscales; 3. joint range of motion (ROM); 4. surgical duration; 5. complications (anterior knee pain, instability and revision rate).

Information sources: PubMed, EMBASE, Cochrane Library, Web of Science, China
Biology Medicine Database (CBM), Wan Fang Database and China National Knowledge Infrastructure Database (CNKI) were searched for the randomized controlled trials (RCTs) meeting prespecified inclusion criteria up to February 1 st, 2021.

Main outcome(s): 1. Knee Society Rating System, clinical (KSCS); 2. functional (KSFS) subscales; 3. joint range of motion (ROM); 4. surgical duration; 5 . complications (anterior knee pain, instability and revision rate).

Quality assessment / Risk of bias analysis: According to the Cochrane Handbook for Systematic Reviews of Interventions, the methodological quality and basis of the included literature were assessed as follows: Random sequence generation, allocation concealment, blinding of participants and personnel, blinding of outcome assessment, incomplete outcome data, selective reporting, and other bias.

Strategy of data synthesis: Statistical Analysis and Data Synthesis were performed with Review Manager Software for Windows (version 5.3). The Standard Mean Difference (SMD) was used to assess continuous outcomes such as pain scores, total opioid consumption, analgesia duration, patient satisfaction, motor strength, and sedation degree with a $95 \%$ confidence interval (Cl). Risk Difference (RD) with a $95 \% \mathrm{Cl}$ were used to assess dichotomous outcomes. The inverse variance and Mantel-Haenszel methods were used to combine separate statistics. If $P$ values were $<0.05$, the results were considered statistically significant. Statistical heterogeneity of the included studies was evaluated using the chi-square test in accordance with the values of 12.12 $<50 \%$ indicated minor heterogeneity between studies, and a fixed-effects model was used to assess the outcomes. 12 between $50 \%$ and $100 \%$ represented substantial heterogeneity and the randomeffects model were used to evaluate these outcomes. subgroup analysis was performed to interpret the potential source of heterogeneity. 
Subgroup analysis: If there is obvious heterogeneity between the included studies, and the heterogeneity will be significantly reduced by dividing into subgroup of different ages, or country, the subgroup analysis will be performed.

Sensitivity analysis: Sensitivity analysis will be used to test the reliability and stability of the meta-analysis results, and to detect the source of heterogeneity. In order to obtain a stable conclusion, we will conduct a sensitivity analysis to eliminate effects of trials with small sample size, eliminate studies not reporting a blind approach to procurement and analysis of data and eliminate studies rated as high risk of bias based on accounting of methodological quality.

Country(ies) involved: China.

Keywords: cruciate-retaining; posteriorstabilized; total knee arthroplasty.

Contributions of each author:

Author 1 - Shiming Zhou.

Email: zhoushiming090@qq.com

Author 2 - Biao Liu.

Author 3 - Jinwei Guo.

Email: ruiying19980618@126.com 